\title{
A Window into Functional Communication: Leveraging Naturalistic Speech Samples in Primary Progressive Aphasia
}

\author{
Jeanne Gallée ${ }^{1 *} \&$ Anna Volkmer ${ }^{2}$ \\ ${ }^{1}$ Program in Speech and Hearing Bioscience and Technology, Harvard University, Cambridge, MA, \\ USA \\ ${ }^{2}$ Division of Psychology and Language Sciences, University College London, London, England, UK \\ * corresponding author: Jeanne Gallée \\ Email: jgallee@g.harvard.edu; ggalleej@gmail.com \\ Address: \\ Jeanne Gallée \\ Harvard Medical School \\ TMEC 235 \\ 260 Longwood Avenue \\ Boston, MA 02115 \\ Phone: +1 (425) 246-5579
}

\section{Conflict of Interest}

There are no relevant conflicts of interest.

\section{Funding}

AV is funded by an NIHR Development Skills Enhancement Award. 


\begin{abstract}
Purpose

Naturalistic speech samples should be routinely collected in the assessment of individuals with communication difficulties. Even when these samples are collected, they are often underutilized. We propose that the analysis of naturalistic speech samples can greatly enhance our understanding and evaluation of the functional impact of primary progressive aphasia (PPA) on communication. First, we review the current practices of evaluating PPA. Second, we provide a framework to optimize the collection, analysis, and interpretation of speech samples to accomplish this goal. In particular, speech samples can be evaluated for measures of informativeness, the presence of atypical patterns of speech, articulatory rate, and pausing, which are all helpful metrics in characterizing disordered speech. These factors can be leveraged to identify both the strengths and difficulties an individual may face in everyday communication.
\end{abstract}

\title{
Conclusion
}

The collection of naturalistic speech in both clinical and naturalistic settings with typical communication partners is highly recommended to best diagnose, monitor and inform treatment plans for individuals with PPA. 


\section{Introduction}

Primary progressive aphasia (PPA) describes a group of neurodegenerative diseases associated with frontotemporal lobar degeneration (FTLD) and Alzheimer's Disease (Gorno-Tempini et al., 2011; Marshall et al., 2018). PPA is characterized by insidious and progressive loss of language. Patients with PPA exhibit variable speech and language deficits as a function of the anatomical sites of maximal atrophy. Three symptomatic profiles of PPA have been established in the literature: nonfluent/agrammatic, semantic, and logopenic (Gorno-Tempini et al., 2011). The primary deficits of the non-fluent variant are agrammatism, effortful speech, and speech sound errors (Gorno-Tempini et al., 2011; Marshall et al., 2018). The core deficits of the semantic variant are anomia and unique deficits to single word-comprehension and object knowledge (Gorno-Tempini et al., 2011; Marshall et al., 2018; Grossman, 2018). Lastly, the primary impairments of the logopenic variant are anomia and impaired repetition ability (Gorno-Tempini et al., 2008; Henry \& Gorno-Tempini, 2010; Gorno-Tempini et al., 2011).

Enormous efforts have been made to standardize the routine assessment of linguistic abilities in PPA (Weintraub et al., 2009; Savage et al., 2013; Sapolsky et al. 2011; Sapolsky et al., 2014; Henry \& Grasso, 2018; Battista et al., 2017; Marshall et al., 2018). Frameworks of disease process for the three variants of PPA (Gorno-Tempini et al., 2011) have been proposed to identify select impairments in domains of articulation, naming, repetition, syntax, semantic knowledge, and comprehension. Importantly, these domains are often assessed in isolation. Nevertheless, up to $30 \%$ of people with PPA do not present with a pattern of impairments that 'fits' one of the three variants (Sajjadi et al., 2012). So, while it seems efficient to characterize selective deficits by domain, these characterizations become more meaningful when we consider their collective contributions to one's communicative ability and impact on quality of life (Ruggero et al., 2019). We must gain a better understanding of how functional 
someone is in their day-to-day interactions and communication. In stroke aphasia, this approach to the assessment of an individual's abilities and difficulties has resulted in the development of effective intervention approaches that target overall communicative effectiveness, rather than focusing on a single domain (Kasselimis et al., 2017; Martin et al., 2008).

In light of the heterogeneity within the PPA population, as well as the diagnostic and access difficulties associated with standardized assessment, we propose that the collection of naturalistic speech samples (in this article we use the term naturalistic speech samples to describe picture descriptions, narratives, discourse and conversation that is captured through audio or video recording) can complement, or even subsume, assessments of isolated domains by allowing us to look at the functional impact of an individuals' strengths and difficulties. Moreover, we believe that measures can be derived from naturalistic speech to help i) determine domain-specific deficits, ii) functionally evaluate speech efficacy, and iii) shape treatment and promote improvements in quality of life related to communication.

\section{A Window into Everyday Exchanges}

Naturalistic speech samples are typically collected in the assessment of communication disorders, but are particularly important in the monitoring of PPA (Sapolsky et al., 2011; Sapolsky et al., 2014; Henry \& Grasso, 2018; Marshall et al., 2018). The ability to identify speech (as captured through measures of articulation and pausing) and language (as captured through naming, repetition, syntax, semantic knowledge, and comprehension) impairment from a routinely collected speech sample is essential in a population with progressive language loss, especially when this type of analysis allows for the unique identification of phenotype-specific impairments that may otherwise be reserved for expensive in vivo imaging or post-mortem analysis. This is not to diminish the utility of standardized assessments that target isolated domains (e.g., picture-word matching, confrontation naming, or the 
repetition of verbalized words and phrases). In fact, these tools are essential to identify key impairments and inform diagnosis. However, if the aim is to determine the extent to which communication is effective, the tools we use must also evaluate functional skills.

Capitalizing on the collection of naturalistic speech samples is a shift towards a more dynamic and "natural" evaluation of our patients' communication needs. The losses that individuals with a diagnosis of PPA face often center on self-expression, agency, and the ability to relate to others (Ash et al., 2006; Ash \& Grossman, 2015; Volkmer et al., 2018; Pozzebon et al., 2018; Ruggero et al., 2019). Thus, the evaluation of speech and language ability must also identify aspects that relate to the use of language and how these aspects may impact functional communication. The approach of addressing the functional components of speech and language production is in line with the therapeutic Life Participation Approach in Aphasia (LPAA; Chapey et al., 2000), which advocates for interventions that focus on and prioritize participation in everyday communication activities. Whilst there is a relatively large body of research literature focusing on the maintenance of speech and language in PPA (Jokel et al, 2014, Croot et al, 2019; Taylor-Rubin, Nickels, \& Croot, 2021), there is a growing focus on compensatory strategy training and life participation to bypass disease-based limitations in PPA. Examples of such include the use of semantic circumlocution (Rogalski et al., 2018; Henry et al., 2019), script training (Schneider et al. 1996; Henry et al., 2018; Henry et al., 2019; Hubbard et al., 2020; Schaffer et al., 2020), enactment (a compensatory strategy where a person is taught to leverage gesture, mime, posture, and prosodic cues to augment communication; Kindell et al., 2013), assistive augmentative communication (Cress \& King, 1999; Fried-Oken, 2008; Fried-Oken et al., 2010; Rogalski et al., 2018; Volkmer et al., 2020), and multimodal communication (Volkmer et al., 2018; Volkmer et al., 2020). Furthermore, a recent review by Volkmer and colleagues (2019) demonstrated that 
interventions that target functional communication are indeed effective for individuals with PPA and essential to improving quality of life (Ruggero et al., 2019).

The richness of naturalistic speech samples allows us to better understand where the breakdowns in communication may occur and thus directly informs long-term care planning and support. The capturing of the relative successes or breakdowns in communication can also directly inform and improve the intervention approach, such as the targeted content and complexity of an alternative augmentative communication device (e.g., a multilayered tablet-based application versus a handmade communication notebook) or the use of strategies for communication partner training (use of gesture versus semantic prompts). This data-driven approach can therefore improve the evaluation and treatment of a person's functional communication. Functional communication is a measure of a person's communicative success in everyday interaction and conversation (Holland, 1982; Crockford \& Lesser, 1994; Armstrong \& Ferguson, 2010; Doedens \& Meteyard, 2019; Volkmer et al., 2020; Gallée et al., 2021). For the purpose of elucidating these considerations, we will present several cases to highlight the complexity of what underlies a "functional" outcome. We provide the following examples to illustrate the ways in which standardized assessments alone cannot adequately characterize a person's communicative effectiveness.

\section{PPA Case Examples}

Consider the case of Bill, a 72-year-old retiree with non-fluent variant PPA (nfvPPA) and relative sparing of motor speech. While agrammatism may reduce the complexity of Bill's sentence production in discourse, he excels at retrieving accurate and content-rich words that are essential to communicate his thoughts. While an assessment of syntax and anomia demonstrates his evident agrammatism, it may be inefficient in capturing his engagement and participation in everyday communication. In fact, the paucity of syntactic complexity of Bill's speech may be compensated for by 
his use of relatively specific and appropriate content words. Furthermore, he supplements his speech with effective use of gestures and facial expressions. If we solely focus on Bill's impairments, we fail to adequately capture meaningful aspects of his overall functional communication (i.e., his ability to participate and compensate in conversation).

For our second example, we present Jada, a 52-year-old mother who received a diagnosis of semantic variant (svPPA) three years ago. Jada's primary symptoms are the loss of word and object knowledge. At first glance, her symptoms easily fly under the radar. Jada participates in small talk with apparent ease, where she routinely shares anecdotes about her morning piano practice. She is observed to describe the music that she plays and the scales she uses to warm up her fingers. However, when asked to point to the piano keys, she responds with a blank stare. Jada's vocabulary becomes increasingly reduced and non-specific and her range of conversational topics more narrow. Her partner and teenage daughter often wonder how much Jada actually understands. They feel she has become selfish and that she doesn't care about their lives. Communication partner training with their clinician helps her husband and daughter understand that her loss of semantic knowledge impacts on her conversation. Naturalistic speech sample analysis also helps to identify stereotyped phrases that characterize her speech when she does not understand what they say, as well as patterns of communication breakdowns. As such, this patient-centered and data-driven approach enables Jada and her family to better communicate.

For our third example, we present the case of Irena, a 48-year-old art teacher diagnosed with mild logopenic variant PPA (lvPPA). Her most apparent symptoms are anomia and repetition, but as the disease remains in the mild stage, her comprehension remains relatively spared. Her daughter notes that the complexity, informativeness, and precision of her speech is reduced. Nevertheless, she regularly expresses her awareness of her word-retrieval difficulties and is most successful when she capitalizes on 
paralinguistic channels, such as facial expressions and vocal inflection to indicate affirmations or dislikes, to enhance conversation. Initially her daughter corrects her errors, resulting in Irena losing confidence which in turn, affects her social life. The pressure for Irena to verbalize is alleviated after her daughter receives guidance to accept her mother's word errors and instead helps Irena and her clinician develop a communication notebook. As a result, Irena is able to meaningfully compensate for her language loss and maintain her relationships and her career. Irena's success in compensating for her symptoms and ability to maintain independence is likely not captured by standardized assessments of confrontation naming or repetition; instead, her agency and functional communication ability are left open to interpretation.

Finally, consider the case of Talbot, a 55-year-old father who has been diagnosed with PPA with symptoms that do not allow for a clear subtype classification. Talbot faces primary deficits in naming and repetition ability, however, unlike individuals with "typical" lvPPA, he presents with striking press of speech. Furthermore, his speech output is pervaded by unintelligible phonemic clusters and jargon. At the milder stages, Talbot may be able to produce targeted content words, measured through an assessment of naming. Unfortunately, the proportion of his unintelligible speech diminishes this relatively preserved capability in everyday conversation. His children find it difficult to parse out his intended messages when he communicates with them in real-time. In contrast, when he calls to leave a message, replaying his voicemails helps them identify what he tried to say.

These examples illustrate that standardized assessments of isolated language domains are insufficient in capturing the ways in which a person can augment their communicative abilities. For example, in the case of Bill, standardized assessment fails to capture his supplemental use of gesture or specificity of speech. While Bill and Irena are good candidates for communication notebooks, Jada and Talbot are not. Even though Jada and Talbot appear relatively fluent in conversation, the extent to which 
they communicate context-appropriate information is relatively low. As such, we believe that the analysis of naturalistic speech samples is dynamic and can greatly enhance the assessment and monitoring of functional speech and language abilities in PPA. Under certain optimal conditions, speech sample analysis may even subsume certain aspects of standardized assessment. This may be particularly relevant if the comprehensiveness of assessment has to be reduced to accommodate patient fatigue or time constraints.

Moreover, the study of naturalistic speech samples in PPA allows us to probe how functionally a person is able to participate in everyday communication or conversational exchanges. In contrast to assessments that require the isolated production or comprehension of single words, assessments of naturalistic speech allow us to contextualize these abilities and create an overall profile of their linguistic capabilities (Ash \& Grossman, 2015). While we are often able to rate the subjective "transactional success” (Ramsberger \& Rende, 2002) and efficiency (Linnik et al., 2016) of a person with aphasia’s communication, there remain gaps in our understanding of how to objectively measure the meaningfulness, appropriateness, and functionality of naturalistic speech (Ramsberger \& Rende, 2002; Linnik et al., 2016). Despite a substantial amount of work investigating connected speech in PPA (Wilson et al., 2010; Ash et al., 2013; Fraser et al., 2014; Boschi et al., 2017; Bonner et al., 2010; Beber et al., 2019a,b; Beales et al., 2018; Berube et al., 2019) and discourse in chronic aphasia (Linnik et al., 2016; Fromm et al., 2017; Berube et al., 2019), objective measurement of task-oriented communication has yet to be established to best predict day-to-day functioning, monitor change, and inform treatment. As all forms of aphasia are often characterized by a reduction in lexical production, we must consider the extent to which an individual is able to functionally communicate (Holland, 1982; Sapolsky et al., 2011; Sapolsky et al., 2014). The role of the clinician then is to establish i) the information an individual 
with PPA can convey, ii) which measures best capture a person's functional communicative ability, and iii) how to best utilize these findings to inform the patient's care.

\section{Optimizing the Conditions of Speech Sample Collection}

Unlike the processes of transcription and analysis, the collection of speech samples is virtually effortless on part of the clinician. However, the conditions under which the samples are collected must be optimized to maximize the potential of the recordings. As such, we recommend that speech samples are recorded in the physical environment of a clinic in the comfort of quiet and sound-proof rooms. To elicit a natural communication environment, we recommend the conversation to be with or to include typical communication partners. Furthermore, the set-up of the room should be comfortable for both the speaker and listener; ideally, the recorder does not have to be moved to capture the speaker's speech during the session. In supplement to audio, we suggest the collection of video recordings to capture body language, such as gesture and eye gaze. Recordings can and should consist of closed standardized assessments, such as picture descriptions or even verbalized reading comprehension, as well as of naturalistic conversation with a typical communication partner. As it may be unnatural to interrupt, the clinician should keep track of the timepoints of assessment and topics of conversation as this will be essential for future analysis and interpretation. In cases where only audio is available, we suggest that the use of gestures should be recorded on a scoresheet as it relates to word-finding difficulty. Moreover, when gesture is used, does it serve to enhance communication? When possible, insight into everyday difficulties can also be captured by recording conversations between the patients and their communication partners - and the ways in which both may benefit from communication strategies and speech therapy (Volkmer et al., 2018).

\section{Analysis of Speech Samples}


Once the speech samples have been collected in optimal conditions, we are prepared to transcribe and analyze our data. Collection of samples at review sessions over time can also allow for the monitoring of progression and severity of disease impact. Transcriptions should include every utterance communicated by the speakers of interest (e.g., the patient). Here we have compiled considerations by subdomain of speech and language faculties to assist the interpretation of speech sample transcriptions.

Articulation Does the number of phonemic substitutions, omissions, and additions render speech widely unintelligible to an uninformed listener? Does the communication partner report difficulty with interpreting the speech of the patient, and does this vary by conversational or environmental contexts?

Production Efficiency Does the number or length of pauses in speech greatly impair the listener's ability to track the intended message? Do these hesitations reduce the speaker's desire to continue verbal communication? Is the patient hindered by failure to initiate communication?

Naming In a controlled paradigm task, such as a picture description, is the individual able to name a sufficient number of targeted open-class words (e.g., nouns, verbs, adjectives, and adverbs)? In instances of failed word retrieval, is the individual able to successfully communicate the intended word through circumlocution by describing semantic features (e.g., function, appearance, location) or implementing gesture and other forms of non-verbal communication? How general (e.g., "thing" or "guy") or specific or frequent (e.g., "abacus") are the words that are produced?

Syntax Is the individual able to produce complete sentences, and if so, what is their grammatical complexity? If this is not the case, which types of closed-class words is the individual omitting? Do hesitations or pauses coincide with failed grammatical endings? While controlled paradigm tasks targeting syntactic constructions may be too foreign or frustrating for individuals to complete, syntactic constructions are easily derived from connected speech. The nature of the task prompt must also be 
considered: a picture description task is likely to evoke an abundance of content words to describe the salient features of the image, whereas an open-ended prompt, such as "What did you do this weekend?" may evoke a response with longer utterances and more opportunities to evaluate syntax.

Comprehension If a natural dialogue between the patient and examiner or communication partner is recorded, how well is the patient able to understand verbal conversation? Is comprehension impacted by phrase length? Is understanding improved through gesture, written information, or the use of physical objects?

\section{Classifying Content in Connected Speech}

Studies of naturalistic speech samples provide insight into speech and language deficits that could inform both restorative and compensatory approaches to treatment. There is a plethora of evidence that the three variants of PPA share a progressive deficit in lexical retrieval, or anomia, as evidenced by performance in tasks ranging from picture naming to a continuous stream of speech, also known as connected speech (Gorno-Tempini et al., 2004; Gorno-Tempini et al., 2011; Rogalski \& Mesulam, 2009; Wilson et al., 2010; Ash et al., 2013; Fraser et al., 2014; Boschi et al., 2017; Bonner et al., 2010; Beber et al., 2019b) and that performance on these measures is reliable, though dependent on task type (Beales et al., 2018). However, the mechanisms by which anomia impacts the lexical content of speech production in PPA, and how this varies by diagnosis, remain to be established. While neither the “transactional success” (Ramsberger \& Rende, 2002) nor efficiency (Linnik et al., 2016) of discourse hinge upon word ordering or selection with fixed precision, both the meaningfulness and appropriateness of a statement rely upon a certain amount of variation (Harris Wright et al., 2003; Gordon, 2008; Linnik et al., 2016) and semantic weight (Linnik et al., 2016) of the words chosen.

Lexical variability has been investigated in the chronic aphasia literature in order to quantify the number of unique words an individual is able to say (Harris Wright et al., 2003; Fergadiotis \& Wright, 
2011; Fergadiotis et al., 2013) as well as the extent to which the content of these utterances is impaired (Berube et al., 2019). In a study of 16 individuals with fluent and non-fluent aphasia, Gordon (2008) determined that the "semantic sufficiency" (p. 840) of a speech sample was directly influenced by the variation of lexical selection, where lower levels of variation significantly correlated with more severe diagnoses of aphasia. Prior investigations of connected speech samples in PPA have reported on the productions of word class, supporting that word class deficits are distinct by subtype. In particular, findings support that people with lvPPA produce fewer nouns and more dysfluencies, such as hesitations and false starts (Wilson et al., 2010, Ash et al., 2013; Boschi et al., 2017), with nfvPPA produce a lower proportion of verbs (Beber et al., 2019a; Wilson et al., 2010; Boschi et al., 2017; Themistocleous et al., 2020), and with svPPA produce fewer nouns (Ash et al., 2013; Fraser et al., 2014; Boschi et al., 2017) and a higher proportion of pronouns (Wilson et al., 2010). Such analyses could be leveraged to relate variability of word choice to the semantic sufficiency of a speech sample and also lead to patientinformed word lists to train in treatment.

\section{Informativeness of Speech}

Beyond lexical output, proportions can also be used to measure the amount of task-relevant speech relative to total output. Recent work by Gallée and colleagues (2021) investigated the overall proportion of informative output in speech samples from individuals with svPPA, nfvPPA, and lvPPA. Informativeness is related to but distinct from meaningfulness. To avoid subjectivity, we will define both terms here. Meaningfulness describes the degree to which a collection of utterances communicates an intended message. Moreover, meaningfulness consists of the overall comprehensibility of a statement. An example of a highly meaningful statement to describe hunger would be "I am hungry and would like to eat." A less grammatically complex yet similarly meaningful statement would be "Food want." Both statements convey that the speaker requires sustenance. In a typical connected speech 
sample of someone with aphasia, many phrases and utterances carry the same level of meaning of the latter statement. We know what the person is intending to say, but it feels impossible to objectively rate the extent to which someone has communicated information or to draw comparisons between individuals. A more objective assessment of information content is informativeness (Doyle, Goda, \& Spencer, 1995). Informativeness is the proportion of information conveyed relative to total output, namely, at the level of correct information units or content units (Doyle et al., 1995; Yorkston \& Beukelman, 1980; Nicholas \& Brookshire, 1993; Agis et al., 2016; Berube et al., 2019; Gallée et al., 2021). While the number of content units a person communicates is open-ended, we can best draw comparisons when we only score content units that are task-specific. For example, in a story retell task, there are characters, objects, actions, and events (content units) that are unambiguously important and specific to the story. For this task, we can conduct group-level comparisons of the proportion of content units a person conveys. In a controlled picture-description task, assessors can systematically pre-identify the essential components of the image using healthy control data to objectively score the raw number of concepts and actions a participant with PPA conveys (see Agis et al., 2016; Berube et al., 2019; Gallée et al., 2021).

In the work by Gallée and colleagues (2021), speech samples of the Western Aphasia BatteryRevised (WAB-R; Kertesz, 2007) "Picnic Scene" task were collected from 31 controls. Of note, the control group was selected to match the PPA groups in terms of age, sex, and education. The transcriptions of these tasks were then analyzed to identify content units (e.g., main concepts, objects, agents, actions, or descriptions) that served to describe aspects of the WAB-R "Picnic Scene." Content units used by at least three controls were compiled into a corpus and categorized by ambiguity and feature type (e.g., object, action, or description; see Table 2 in Gallée et al., 2021 for the exact corpus). As a content unit represents a concept or aspect of the picture, synonyms were clustered (e.g., the 
content unit for the house in the WAB-R "Picnic Scene" includes all appropriate referents, such as "home," "cottage," and “cabin").

This corpus was then used to score the transcripts collected from 25 nfvPPA, 19 svPPA, and 26 lvPPA. When a content unit occurred multiple times, only the first instance was scored. This procedure allowed the researchers to identify the amount of unique information a person conveyed. The raw scores were then converted into normalized scores to reflect the relative proportion of content units to the total number of utterances (words, filler words, false starts): $\frac{\text { Raw Number of Content Units }}{\text { Total Number of Utterances }}$ This objective score captures the relative informativeness (Doyle et al., 1995) of a speech sample given specific taskconstraints (i.e. a picture description). Examples of transcriptions coded for informativeness are included below. The bracketed numbers represent the content units identified in Table 2 of Gallée and colleagues (2021).

1. "Two people [21] have gone out and decided to have a picnic [7]. One of them is reading [36] his book [14] the other one is unwrapping a sandwich [63]. I think. It is a lovely [41] day [58] . There is a lovely [41] house [2] lovely scenery [44] a tree [15] in the background [64a] with a lovely house. They are also on some kind of- they are also on a lake [10] with a sailboat [6] and another younger [55] person a little [55a] boy [11] is flying [34] a kite [1] and wearing [53a] shorts [49] and having fun [38] with this dog [4] chasing [39] him and in the background we also have someone [45d] in there looks like they're fishing [33] and maybe on the boat out fishing."

2. "Well, there is a picnic [7] going on, a man [43a] and a woman [8] uh are on a blanket [25] and they have a radio [22] on, I guess, the antenna is up, uh, and the man is reading [36] a book [14] with the glasses [31] and uh the woman is pouring [37] a drink [3] from a bottle [32] uh and the 
uh there is a picnic basket [30] on the uh blanket [25] between the man and the woman and there is a uh the man has taken his sandals [19] off, and are on the lawn near a lake [10] uh and there is a boy [11] going by flying [34] a kite [1] with a dog [4] at his heels [39], and uh beach [5] out front [64] and a girl [12] building sandcastles [9], yeah, and uh, there is a dock [13] jutting out from the beach where a mans [43b] with a hat on is catching [323] a- a fish [20]and a sailboat [6] going by on the lake [10], four-seventy is on the sail, there are two people [21] on the boat, and there is a house [2], set back [64a] uh from the lake [10] with a tree [15] in front uh uh sichuous tree in the front of the house".

The researchers found that the informativeness of the control and nfvPPA groups did not differ from one another whereas that of the svPPA and lvPPA groups was significantly reduced. Importantly, the nfvPPA transcripts were shorter than those of the other three groups. Nevertheless, the nfvPPA group produced a larger number of raw content units (non-normalized measure) as well as greater informativeness (normalized measure) than svPPA and lvPPA. This finding suggests that content unit analysis of speech samples can help differentiate nfvPPA from the other two variants.

It is important to note that this methodology can only be applied to tasks where there are clear goals. In the case of a picture description, there are clear elements of the picture that must be named or described for the speech sample to be rated as task-relevant and informative. Procedural tasks (e.g., "Tell me how to make a peanut butter sandwich) and fairytale retells also have clear goals for both the speaker and examiner. Of note, procedurals and story retell tasks rely upon generational, cultural, and linguistic knowledge that is not ubiquitously shared across communities (hint: use and interpret with caution!). For other more open-ended prompts (e.g., "What did you do this weekend?"), a more participant-specific approach may be warranted. For example, the communication partner of the patient 
may be interviewed about their past weekend so that the examiner knows which details the patient could describe. This latter form of naturalistic speech analysis is more reflective of everyday conversation but also requires a more subjective interpretation as it cannot be compared to normative data. Recordings of the person speaking with their communication partner can in turn also illuminate how the partner deals with a potential lack of informativeness. This can provide a basis for development of compensatory strategies for the person with PPA, and education and communication training for the partner.

\section{Quantitative Rating of Atypical Patterns of Speech}

The process of transcribing naturalistic speech samples can be effortful and time-consuming without a clear end-goal or analysis plan. However, we believe that these transcriptions provide direct insight into how functional a person's communicative ability is and can greatly inform the source of breakdowns in communication. Furthermore, they allow us to disentangle factors of informativeness and fluency. In recent work by Gallée and colleagues (2021), two methods were implemented to assess the source of breakdowns in communication in PPA. This work was motivated by three questions: (1) Is the amount of information conveyed in a picture description task reduced in individuals with mild PPA?; (2) Is the informativeness of speech reduced by atypical patterns of speech?; (3) Do the three variants of PPA exhibit different atypical speech patterns and functional communication abilities? Such differences are often discussed among clinicians and investigators, but have not received sufficient attention in the prior literature.

To investigate these questions, the researchers evaluated the 70 aforementioned transcriptions for the presence of five atypical speech patterns. The patterns of interest were i) self-referential (e.g., "My grand-daughter also plays on the beach"), ii) statements about reduced performance (e.g., "Don’t know that word" or "I should know that"), iii) tangential (e.g., "She shouldn't be doing that"), iv) "empty" speech (e.g., "Uh yes, oh uh"), and iv) false starts (e.g., " $Y$ - $y$ - $y$ - you”). The raters of the transcripts were 
blinded to the diagnoses of the patient to reduce potential biases. These patterns were all determined to not be task-relevant and uninformative, and thus, detrimental to functional communication.

Similar to the informativeness analysis, Gallée and colleagues' (2021) found that the transcripts of the control and nfvPPA groups did not differ for any of the five patterns. Comparatively, the svPPA and lvPPA transcripts included significantly more atypical patterns of speech. This finding suggests that the nfvPPA patients are more efficient in their production of content than people with svPPA and lvPPA, despite the relative paucity of their output. Importantly, the causes of communicative breakdowns in lvPPA and svPPA were believed to be distinct from one another also. While both groups were found to often comment on their reduced performance, the lvPPA transcripts contained significantly more empty speech and false starts. In contrast, the svPPA transcriptions contained significantly more self-referential statements, which often serve to mask anomia or comprehension difficulties.

As such, functional communication ability differed between variants. Furthermore, for svPPA and lvPPA, the qualitative nature of reduced informativeness differed. While the latter group's speech contained many more instances of empty speech and false starts, the true words that were produced were more likely to be task-specific. On the surface, svPPA transcripts appeared more fluent. Upon closer inspection of the actual content, the proportion of task-relevant - or situationally functional - output was rather low. These are important distinctions that only analyses of naturalistic speech can provide.

\section{Operationalizing Fluency Through Measures of Articulatory Rate and Pausing}

Fluency is a traditional measure used in aphasia to distinguish the "fluent" from the "non-fluent" diagnoses. Fluency often refers to the efficiency at which speech is articulated and relies upon processes of retrieval, formulation, and production (Gordon \& Clough, 2020). In addition to speech content,

articulatory rate $\left(\frac{\text { Total Number of Syllables }}{\text { Total Duration of Speech }}\right)$ and pause analysis can be used to phenotype PPA (see 
Cordella et al., 2017, 2019). As the primary symptoms of nfvPPA include effortful speech production and syntactic impairment, nfvPPA speech is often considered to be slower than that of lvPPA and svPPA. Recent work by Cordella and colleagues (2017) found that the articulatory rate of nfvPPA was significantly slower than the other two variants and half that of controls. Furthermore, the researchers found that the average pause length for nfvPPA hovered between 1 and 1.5 seconds. In contrast, the pauses for svPPA and lvPPA were significantly shorter and between 0.5 and 1 seconds. This is consistent with work from Ballard and colleagues (2014), who showed that the median silence duration of individuals with nfvPPA was almost twice that of lvPPA, and approximately triple that of healthy controls. These findings support that pauses and word-finding hesitations have a quantifiable impact on speech and its perceived efficiency. While a person may maintain general meaningfulness or taskrelevant informativeness in communication, greater pauses between utterance production are i) inefficient, ii) overly taxing on the interlocutor, and iii) may result in the speaker refraining from verbal communication. As such, an essential consideration for speech and language therapy is the functional impact of perceived fluency on a speaker's communication (a metric that is only possible through naturalistic speech sample analysis and not through traditional standardized assessments) and whether options such as alternative augmentative communication (AAC) or communication partner training may be helpful considerations. AAC can enable the person with PPA to express themselves (Fried-Oken, 2008; Fried-Oken, Rowland, \& Gibbons, 2010) and Communication Partner Training can provide much sought-after practical strategies for the people around the person with PPA to maximize the flow of conversation (Volkmer et al, 2021).

\section{Gesture Use in PPA}

There is evidence that people with aphasia enhance their communication by using gesture during more complex language tasks (Kita \& Davies, 2009), particularly in descriptions containing highly 
imageable content (Hostetter \& Alibali, 2019). Synthesizing work by de Ruiter and de Beer (2013) suggests that gesture is used efficiently and in place of words and phrases by people with aphasia. Furthermore, work by Kindell and colleagues (2013) demonstrates that people with svPPA are able to effectively use whole body gestures and mime to convey narrative. Our understanding of the ways in which gesture can facilitate language production is currently limited (Rose et al., 2013), however, can and must be included in our framework of functional communication. In the instance where our Irena is unable to retrieve the word "teapot", would we not consider the motion of pouring tea to be a successful and functional use of multimodal communication?

\section{Conclusion}

Evaluation and monitoring of PPA can be greatly enhanced through the assessment of naturalistic speech samples through audio and/or video recording. The richness of naturalistic speech allows for both quantitative and qualitative analyses that can illustrate how functionally an individual is able to linguistically participate. Moreover, we believe that to best predict and facilitate an individual's participation in communication, it is essential to conduct multidimensional assessment that reflects dayto-day speech production. For successful data collection, both constrained and open-ended speecheliciting tasks should be collected to capture the range of conversational abilities and include their relevant communication partners where possible. Our proposal here is inspired by the goals of LPAA and improving the quality of care people with PPA receive. Naturalistic speech samples contain a wealth of data that can be leveraged to better understand, monitor, and treat the nature of communicative impairment in PPA. Here, we highlight the importance of the routine collection of naturalistic speech samples to identify and monitor impairments as this serves to i) capture impairments unique to the individual assessed, ii) builds upon the growing shift to examine the interplay of language functions, 
rather than the isolated parts, and iii) capture direct insight into the individual's ability to communicate in everyday conversation: functional communication.

\section{Acknowledgments}

We gratefully acknowledge the countless contributions of our patients and families. This work would not be possible without them. We would also like to thank Victoria Tilton-Bolowsky for her essential revisions to this manuscript.

\section{References}

1. Agis, D., Goggins, M. B., Oishi, K., Oishi, K., Davis, C., Wright, A., ... \& Hillis, A. E. (2016). Picturing the size and site of stroke with an expanded National Institutes of Health Stroke Scale. Stroke, 47(6), 1459-1465. https://doi.org/10.1161/STROKEAHA.115.012324

2. Ash, S., Moore, P., Antani, S., McCawley, G., Work, M., and Grossman, M. (2006). Trying to tell a tale. Discourse impairments in progressive aphasia and frontotemporal dementia. Neurology, 66, 1405-1413. https://doi.org/10.1212/01.wnl.0000210435.72614.38)

3. Ash, S., Evans, E., O'Shea, J., Powers, J., Boller, A., Weinberg, D., ... \& Grossman, M. (2013). Differentiating primary progressive aphasias in a brief sample of connected speech. Neurology, 81(4), 329-336. https://doi.org/10.1212/WNL.0b013e31829c5d0e

4. Ash, S., and Grossman, M. (2015). "Why study connected speech production?," in Cognitive Neuroscience of Natural Language Use, ed R. M. Willems (Cambridge: Cambridge University Press), 29-58.

5. Ballard, K. J., Savage, S., Leyton, C. E., Vogel, A. P., Hornberger, M., \& Hodges, J. R. (2014). Logopenic and nonfluent variants of primary progressive aphasia are differentiated by acoustic 
measures of speech production. PloS one, 9(2), e89864.

https://doi.org/10.1371/journal.pone.0089864

6. Battista, P., Miozzo, A., Piccininni, M., Catricalà, E., Capozzo, R., Tortelli, R., ... \& Logroscino, G. (2017). Primary progressive aphasia: a review of neuropsychological tests for the assessment of speech and language disorders. Aphasiology, 31(12), 1359-1378.

https://doi.org/10.1080/02687038.2017.1378799

7. Beales, A., Cartwright, J., Whitworth, A., \& Panegyres, P. K. (2016). Exploring generalisation processes following lexical retrieval intervention in primary progressive aphasia. International Journal of Speech-Language Pathology, 18(3), 299-314.

https://doi.org/10.3109/17549507.2016.1151936

8. Beales, A., Whitworth, A., Cartwright, J., Panegyres, P. K., \& Kane, R. T. (2018). Determining stability in connected speech in primary progressive aphasia and Alzheimer's disease.

International Journal of Speech-Language Pathology, 20(3), 361-370.

https://doi.org/10.1080/17549507.2018.1442498

9. Beber, B. C., Mandelli, M. L., Santos, M. A. S., Binney, R. J., Miller, B., Chaves, M. L., ... \& Shapiro, K. A. (2019a). A behavioral study of the nature of verb-noun dissociation in the nonfluent variant of primary progressive aphasia. Aphasiology, 33(2), 200-215. https://doi.org/10.1080/02687038.2018.1461799

10. Berube, S., Nonnemacher, J., Demsky, C., Glenn, S., Saxena, S., Wright, A., ... \& Hillis, A. E. (2019). Stealing cookies in the twenty-first century: measures of spoken narrative in healthy versus speakers with aphasia. American journal of speech-language pathology, 28(1S), 321-329. https://doi.org/10.1044/2018_AJSLP-17-0131 
11. Bonner, M. F., Ash, S., \& Grossman, M. (2010). The new classification of primary progressive aphasia into semantic, logopenic, or nonfluent/agrammatic variants. Current neurology and neuroscience reports, 10(6), 484-490. https://DOI.ORG/10.1007/s11910-010-0140-4

12. Boschi, V., Catricala, E., Consonni, M., Chesi, C., Moro, A., \& Cappa, S. F. (2017). Connected speech in neurodegenerative language disorders: a review. Frontiers in psychology, 8, 269. https://doi.org/10.3389/fpsyg.2017.00269

13. Chapey, R., Duchan, J. F., Elman, R. J., Garcia, L. J., Kagan, A., Lyon, J. G., \& Simmons Mackie, N. (2000). Life participation approach to aphasia: A statement of values for the future. The ASHA Leader, 5(3), 4-6. https://doi.org/10.1044/leader.FTR.05032000.4

14. Cordella, C., Dickerson, B. C., Quimby, M., Yunusova, Y., \& Green, J. R. (2017). Slowed articulation rate is a sensitive diagnostic marker for identifying non-fluent primary progressive aphasia. Aphasiology, 31(2), 241-260. https://doi.org/10.1080/02687038.2016.1191054

15. Cordella, C., Quimby, M., Touroutoglou, A., Brickhouse, M., Dickerson, B. C., \& Green, J. R. (2019). Quantification of motor speech impairment and its anatomic basis in primary progressive aphasia. Neurology, 92(17), e1992-e2004. https://doi.org/10.1212/WNL.0000000000007367

16. Cress, C., \& King, J. (1999). AAC strategies for people with primary progressive aphasia without dementia: Two case studies. Augmentative and Alternative Communication, 15(4), 248259. https://doi.org/10.1080/07434619912331278785

17. Crockford, C., \& Lesser, R. (1994). Assessing functional communication in aphasia: Clinical utility and time demands of three methods. International Journal of Language \& Communication Disorders, 29(2), 165-182. https://doi.org/10.3109/13682829409041490 
18. Croot, K., Raiser, T., Taylor-Rubin, C., Ruggero, L., Ackl, N., Wlasich, E., ... \& Nickels, L. (2019). Lexical retrieval treatment in primary progressive aphasia: An investigation of treatment duration in a heterogeneous case series. Cortex, 115, 133-158.

https://doi.org/10.1016/j.cortex.2019.01.009

19. De Ruiter, J. P., \& de Beer, C. (2013). A critical evaluation of models of gesture and speech production for understanding gesture in aphasia. Aphasiology, 27(9), 1015-1030. https://doi.org/10.1080/02687038.2013.797067

20. Doyle, P. J., Goda, A. J., \& Spencer, K. A. (1995). The communicative informativeness and efficiency of connected discourse by adults with aphasia under structured and conversational sampling conditions. American Journal of Speech-Language Pathology, 4(4), 130-134. https://doi.org/10.1044/1058-0360.0404.130

21. Fergadiotis, G., \& Wright, H. H. (2011). Lexical diversity for adults with and without aphasia across discourse elicitation tasks. Aphasiology, 25(11), 1414-1430. https://doi.org/10.1080/02687038.2011.603898

22. Fergadiotis, G., Wright, H. H., \& West, T. M. (2013). Measuring lexical diversity in narrative discourse of people with aphasia. American journal of speech-language pathology, 22(2), S397S408. https://doi.org/10.1044/1058-0360(2013/12-0083)

23. Fraser, K. C., Meltzer, J. A., Graham, N. L., Leonard, C., Hirst, G., Black, S. E., \& Rochon, E. (2014). Automated classification of primary progressive aphasia subtypes from narrative speech transcripts. Cortex, 55, 43-60. https://doi.org/10.1016/j.cortex.2012.12.006

24. Fried-Oken, M. (2008). Augmentative and alternative communication treatment for persons with primary progressive aphasia. Perspectives on Augmentative and Alternative Communication, 17(3), 99-104. https://doi.org/10.1044/aac17.3.99 
25. Fried-Oken, M., Rowland, C., \& Gibbons, C. (2010). Providing augmentative and alternative communication treatment to persons with progressive nonfluent aphasia. Perspectives on Neurophysiology and Neurogenic Speech and Language Disorders, 20(1), 21-25. https://doi.org/10.1044/nnsld20.1.21

26. Fromm, D., Forbes, M., Holland, A., Dalton, S. G., Richardson, J., \& MacWhinney, B. (2017). Discourse characteristics in aphasia beyond the Western Aphasia Battery cutoff. American Journal of Speech-Language Pathology, 26(3), 762-768. https://doi.org/10.1044/2016_AJSLP$16-0071$

27. Gallée, J., Cordella, C., Fedorenko, E., Hochberg, D., Touroutoglou, A., Quimby, M., \& Dickerson, B. C. (2021). Breakdowns in Informativeness of Naturalistic Speech Production in Primary Progressive Aphasia. Brain Sciences, 11(2), 130. https://doi.org/10.3390/brainsci11020130

28. Gordon, J. K. (2008). Measuring the lexical semantics of picture description in aphasia. Aphasiology, 22(7-8), 839-852. https://doi.org/10.1080/02687030701820063

29. Gordon, J. K., \& Clough, S. (2020). How fluent? Part B. Underlying contributors to continuous measures of fluency in aphasia. Aphasiology, 34(5), 643-663. https://doi.org/10.1080/02687038.2020.1712586

30. Gorno-Tempini, M. L., Dronkers, N. F., Rankin, K. P., Ogar, J. M., Phengrasamy, L., Rosen, H. J., . . Miller, B. L. (2004). Cognition and anatomy in three variants of primary progressive aphasia. Annals of Neurology: Official Journal of the American Neurological Association and the Child Neurology Society, 55(3), 335-346. https://doi.org/10.1002/ana.10825 
31. Gorno-Tempini, M. L., Brambati, S. M., Ginex, V., Ogar, J., Dronkers, N. F., Marcone, A., .. . Miller, B. L. (2008). The logopenic/phonological variant of primary progressive aphasia. Neurology, 71(16), 1227-1234. https://doi.org/10.1212/01.wnl.0000320506.79811.da

32. Gorno-Tempini, M. L., Hillis, A. E., Weintraub, S., Kertesz, A., Mendez, M., Cappa, S. F., . . Boeve, B. F. (2011). Classification of primary progressive aphasia and its variants. Neurology, 76(11), 1006-1014. https://doi.org/10.1212/WNL.0b013e31821103e6

33. Henry, M. L., \& Gorno-Tempini, M. L. (2010). The logopenic variant of primary progressive aphasia. Current opinion in neurology, 23(6), 633. https://doi.org/10.1097/WCO.0b013e32833fb93e

34. Henry, M. L., \& Grasso, S. M. (2018). Assessment of Individuals with Primary Progressive Aphasia. Seminars in speech and language, 39(3), 231-241. https://doi.org/10.1055/s-00381660782

35. Henry, M. L., Hubbard, H. I., Grasso, S. M., Mandelli, M. L., Wilson, S. M., Sathishkumar, M. T., ... \& Gorno-Tempini, M. L. (2018). Retraining speech production and fluency in nonfluent/agrammatic primary progressive aphasia. Brain, 141(6), 1799-1814. https://doi.org/10.1093/brain/awy149

36. Henry, M. L., Hubbard, H. I., Grasso, S. M., Dial, H. R., Beeson, P. M., Miller, B. L., \& GornoTempini, M. L. (2019). Treatment for word retrieval in semantic and logopenic variants of primary progressive aphasia: Immediate and long-term outcomes. Journal of Speech, Language, and Hearing Research, 62(8), 2723-2749. https://doi.org/10.1044/2018_JSLHR-L-18-0144

37. Holland, A. L. (1982). Observing functional communication of aphasic adults. Journal of Speech and Hearing Disorders, 47(1), 50-56. 
38. Hostetter, A. B., \& Alibali, M. W. (2019). Gesture as simulated action: Revisiting the framework. Psychonomic bulletin \& review, 26(3), 721-752. https://doi.org/10.3758/s13423-018$1548-0$

39. Hubbard, H. I., Nelson, L. A., \& Richardson, J. D. (2020, January). Can Script Training Improve Narrative and Conversation in Aphasia across Etiology?. In Seminars in speech and language (Vol. 41, No. 01, pp. 099-124). Thieme Medical Publishers. https://doi.org/10.1055/s0039-3401030

40. Jokel, R., Graham, N. L., Rochon, E., \& Leonard, C. (2014). Word retrieval therapies in primary progressive aphasia. Aphasiology, 28(8-9), 1038-1068. https://doi.org/10.1080/02687038.2014.899306

41. Kasselimis, D. S., Simos, P. G., Peppas, C., Evdokimidis, I., \& Potagas, C. (2017). The unbridged gap between clinical diagnosis and contemporary research on aphasia: A short discussion on the validity and clinical utility of taxonomic categories. Brain and language, 164, 63-67. https://doi.org/10.1016/j.bandl.2016.10.005

42. Kindell, J., Sage, K., Keady, J., \& Wilkinson, R. (2013). Adapting to conversation with semantic dementia: using enactment as a compensatory strategy in everyday social interaction. International Journal of Language \& Communication Disorders, 48(5), 497-507.

43. Kita, S., \& Davies, T. S. (2009). Competing conceptual representations trigger co-speech representational gestures. Language and Cognitive Processes, 24(5), 761-775. https://doi.org/10.1080/01690960802327971

44. Linnik, A., Bastiaanse, R., \& Höhle, B. (2016). Discourse production in aphasia: A current review of theoretical and methodological challenges. Aphasiology, 30(7), 765-800. https://doi.org/10.1080/02687038.2015.1113489 
45. Marshall, C. R., Hardy, C. J., Volkmer, A., Russell, L. L., Bond, R. L., Fletcher, P. D., ... \& Fox, N. C. (2018). Primary progressive aphasia: a clinical approach. Journal of Neurology, 265(6), 1474-1490. https://doi.org/10.1007/s00415-018-8762-6

46. Nicholas, L. E., \& Brookshire, R. H. (1993). A system for quantifying the informativeness and efficiency of the connected speech of adults with aphasia. Journal of Speech, Language, and Hearing Research, 36(2), 338-350.

47. Pozzebon, M., Douglas, J., \& Ames, D. (2018). Facing the challenges of primary progressive aphasia: The spousal perspective. Journal of Speech, Language, and Hearing Research, 61(9), 2292-2312. https://doi.org/10.1044/2018_JSLHR-L-17-0492

48. Ramsberger, G., \& Rende, B. (2002). Measuring transactional success in the conversation of people with aphasia. Aphasiology, 16(3), 337-353. https://doi.org/10.1080/02687040143000636

49. Rogalski, E. J., \& Mesulam, M. M. (2009). Clinical trajectories and biological features of primary progressive aphasia (PPA). Current Alzheimer Research, 6(4), 331-336. https://doi.org/10.2174/156720509788929264

50. Rogalski, E. J., \& Khayum, B. (2018, July). A life participation approach to primary progressive aphasia intervention. In Seminars in speech and language (Vol. 39, No. 3, p. 284). NIH Public Access. https://doi.org/10.1055/s-0038-1660786

51. Rose, M. L., Raymer, A. M., Lanyon, L. E., \& Attard, M. C. (2013). A systematic review of gesture treatments for post-stroke aphasia. Aphasiology, 27(9), 1090-1127. https://doi.org/10.1080/02687038.2013.805726

52. Ruggero, L., Nickels, L., \& Croot, K. (2019). Quality of life in primary progressive aphasia: What do we know and what can we do next?. Aphasiology, 33(5), 498-519. https://doi.org/10.1080/02687038.2019.1568135 
53. Sajjadi, S. A., Patterson, K., Arnold, R. J., Watson, P. C., \& Nestor, P. J. (2012). Primary progressive aphasia: a tale of two syndromes and the rest. Neurology, 78(21), 1670-1677. https://doi.org/10.1212/WNL.0b013e3182574f79

54. Sapolsky, D., K. Domoto-Reilly, A. Negreira, M. Brickhouse, S. M. McGinnis and B. C. Dickerson (2011). "Monitoring progression of primary progressive aphasia: Current approaches and future directions." Neurodegenerative Disease Management 1(1): 43-55. https://doi.org/10.2217/nmt.11.2

55. Sapolsky, D., K. Domoto-Reilly and B. C. Dickerson (2014). "Use of the Progressive Aphasia Severity Scale (PASS) in monitoring speech and language status in PPA." Aphasiology 28(8-9): 993-1003. PMC4235969. https://doi.org/10.1080/02687038.2014.931563

56. Savage, S. A., Ballard, K. J., Piguet, O., \& Hodges, J. R. (2013). Bringing words back to mindImproving word production in semantic dementia. Cortex, 49(7), 1823-1832. https://doi.org/10.1016/j.cortex.2012.09.014

57. Schaffer, K. M., Wauters, L., Berstis, K., Grasso, S. M., \& Henry, M. L. (2020). Modified script training for nonfluent/agrammatic primary progressive aphasia with significant hearing loss: A single-case experimental design. Neuropsychological Rehabilitation, 1-30. https://doi.org/10.1080/09602011.2020.1822188

58. Schneider, S. L., Thompson, C. K., \& Luring, B. (1996). Effects of verbal plus gestural matrix training on sentence production in a patient with primary progressive aphasia. Aphasiology, 10(3), 297-317. https://doi.org/10.1080/02687039608248414

59. Taylor-Rubin, C., Nickels, L., \& Croot, K. (2021). Exploring the effects of verb and noun treatment on verb phrase production in primary progressive aphasia: A series of single case 
experimental design studies. Neuropsychological Rehabilitation, 1-43.

https://doi.org/10.1080/09602011.2021.1879174

60. Themistocleous, C., Webster, K., Afthinos, A., \& Tsapkini, K. (2020). Part of speech production in patients with Primary Progressive Aphasia: An analysis based on natural language processing. American Journal of Speech-Language Pathology, 1-15. https://doi.org/10.1044/2020_AJSLP-19-00114

61. Martin, N., Thompson, C. K., \& Worrall, L. (2007). Aphasia rehabilitation: The impairment and its consequences. Plural Publishing.

62. Volkmer, A., Spector, A., Warren, J. D., \& Beeke, S. (2018). The 'Better Conversations with Primary Progressive Aphasia (BCPPA)'program for people with PPA (Primary Progressive Aphasia): protocol for a randomised controlled pilot study. Pilot and feasibility studies, 4(1), 110. https://doi.org/10.1186/s40814-018-0349-6

63. Volkmer, A., A. Spector, V. Meitanis, J. D. Warren and S. Beeke (2020). Effects of functional communication interventions for people with primary progressive aphasia and their caregivers: a systematic review. Aging Ment Health 24(9), 1381-1393.

https://doi.org/10.1080/13607863.2019.1617246

64. Volkmer, A., Spector, A., Swinburn, K., Warren, J. D., \& Beeke, S. (2021). Using the Medical Research Council Framework for the development of a communication partner training intervention for people with primary progressive aphasia (PPA): Better Conversations with PPA. https://doi.org/10.21203/rs.3.rs-228838/v1

65. Weintraub, S., Mesulam, M. M., Wieneke, C., Rademaker, A., Rogalski, E. J., \& Thompson, C. K. (2009). The northwestern anagram test: measuring sentence production in primary 
progressive aphasia. American Journal of Alzheimer's Disease \& Other Dementias®, 24(5), 408416. https://doi.org/10.1177/1533317509343104

66. Wilson, S. M., Henry, M. L., Besbris, M., Ogar, J. M., Dronkers, N. F., Jarrold, W., ... \& GornoTempini, M. L. (2010). Connected speech production in three variants of primary progressive aphasia. Brain, 133(7), 2069-2088. https://doi.org/10.1093/brain/awq129

67. Harris Wright, H., Silverman, S., \& Newhoff, M. (2003). Measures of lexical diversity in aphasia. Aphasiology, 17(5), 443-452. https://doi.org/10.1080/02687030344000166

68. Yorkston, K. M., \& Beukelman, D. R. (1980). An analysis of connected speech samples of aphasic and normal speakers. Journal of speech and hearing disorders, 45(1), 27-36. https://doi.org/10.1044/jshd.4501.27 